\title{
BIT ERROR RATE PERFORMANCE OF A FREE SPACE OPTICAL LINK USING DOUBLE CLAD FIBERS
}

\author{
Joseph Sullivan \\ University of Oklahoma \\ Bryan Schoenholz and Sarah Tedder \\ NASA Glenn Research Center
}




\section{Objective}

Determine bit error rate (BER) performance in a passively aligned free space optical link (FSOL) utilizing Double clad fibers (DCF) for transmitting and receiving, demonstrating the viability of a symmetric bidirectional FSOL using DCF.

\section{Background}

Small form-factor pluggable (SFP) transceivers are a low cost, commercial off the shelf (COTS) implementation of a high data rate free space optical link (FSOL). Long range SFPs are designed for fiber optic systems using single mode fibers (SMF).

Typical Bi-directional FSOL

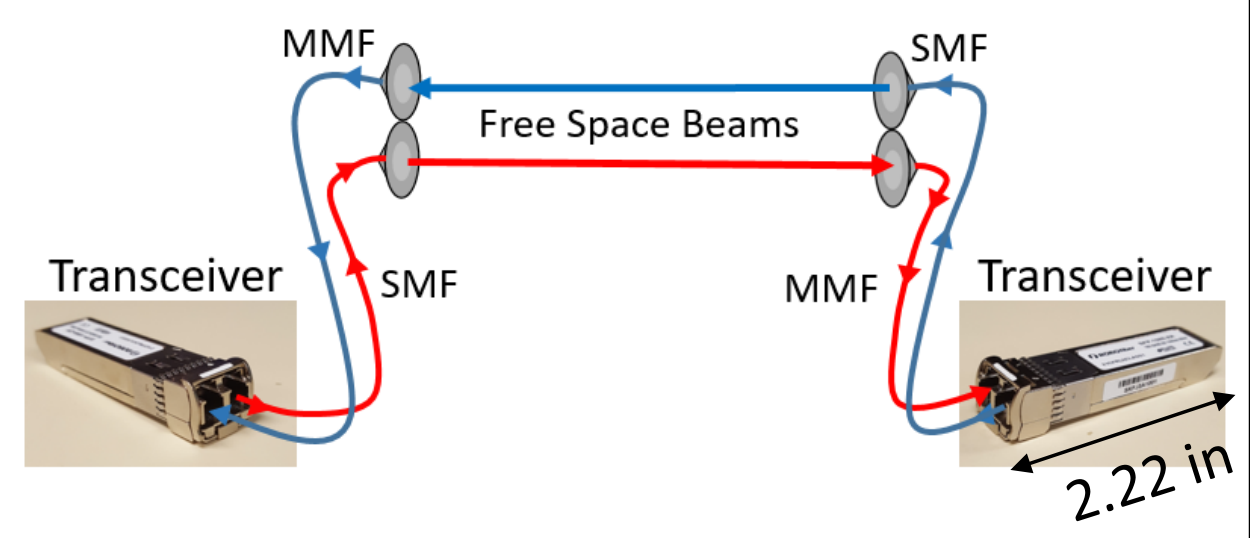

Bi-directional FSOL using DCF

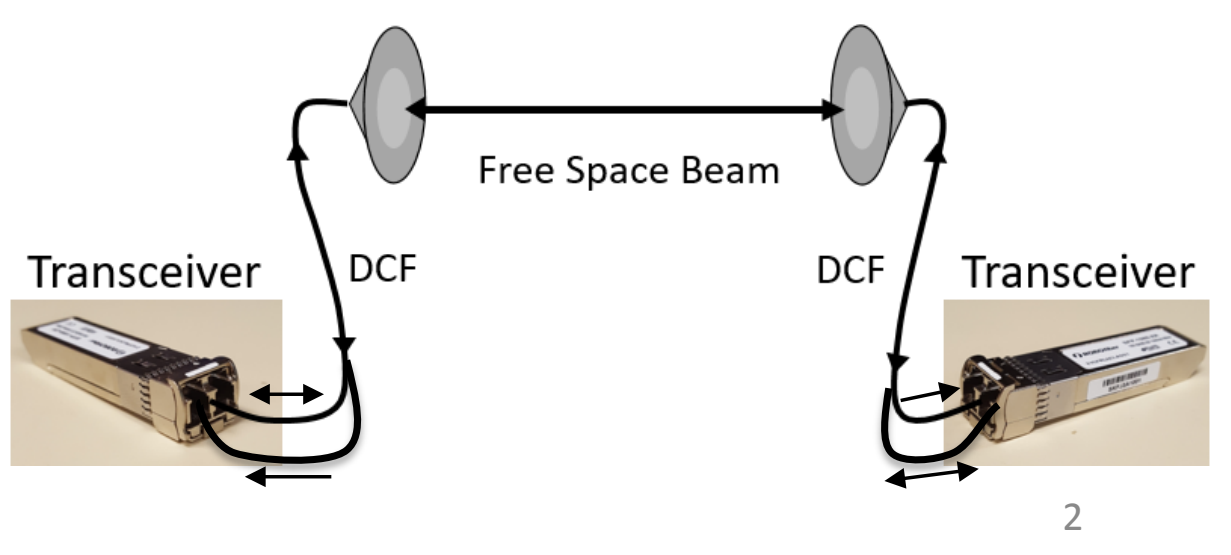




\section{Background}

\section{Fibers}

- $\quad$ Large core multi-mode receiving fibers (MMF) improve misalignment tolerance

- Using MMF to transmit causes power instability in laser beam

- Double clad fibers (DCF) can transmit a stable Gaussian beam through the single mode core and receive in the large inner cladding

- DCF enable a single bidirectional optical path with symmetric transmit and receive setups allowing for low size, weight, and power (SWaP)

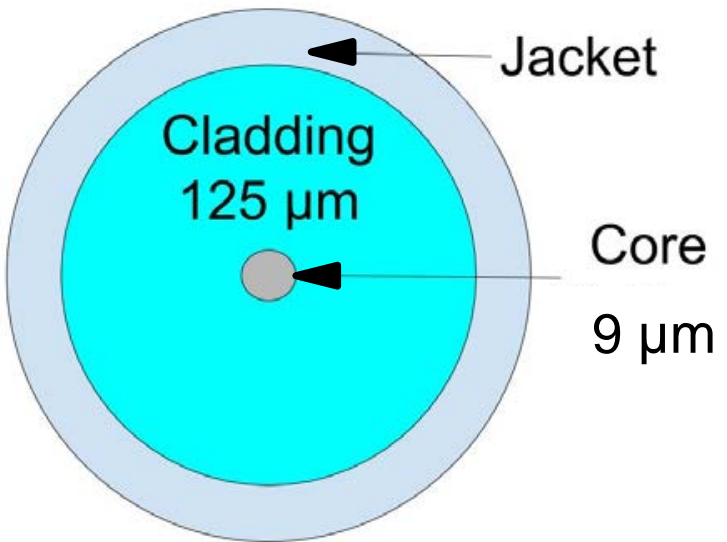

$9 \mu \mathrm{m}$ Single Mode Fiber

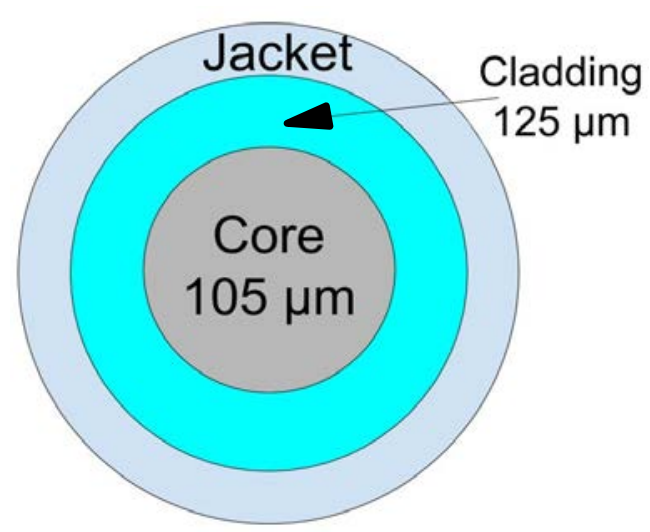

$105 \mu \mathrm{m}$ Multi Mode Fiber

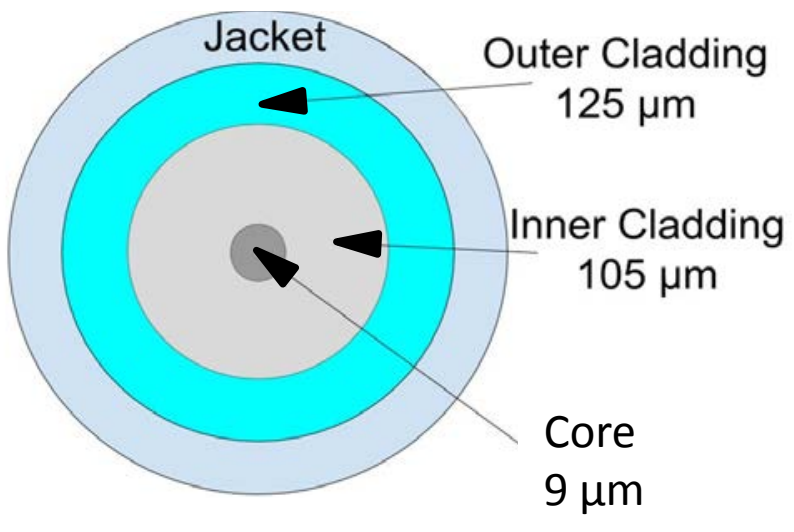

9/105 $\mu \mathrm{m}$ Double Clad Fiber 


\section{DCF Coupler}

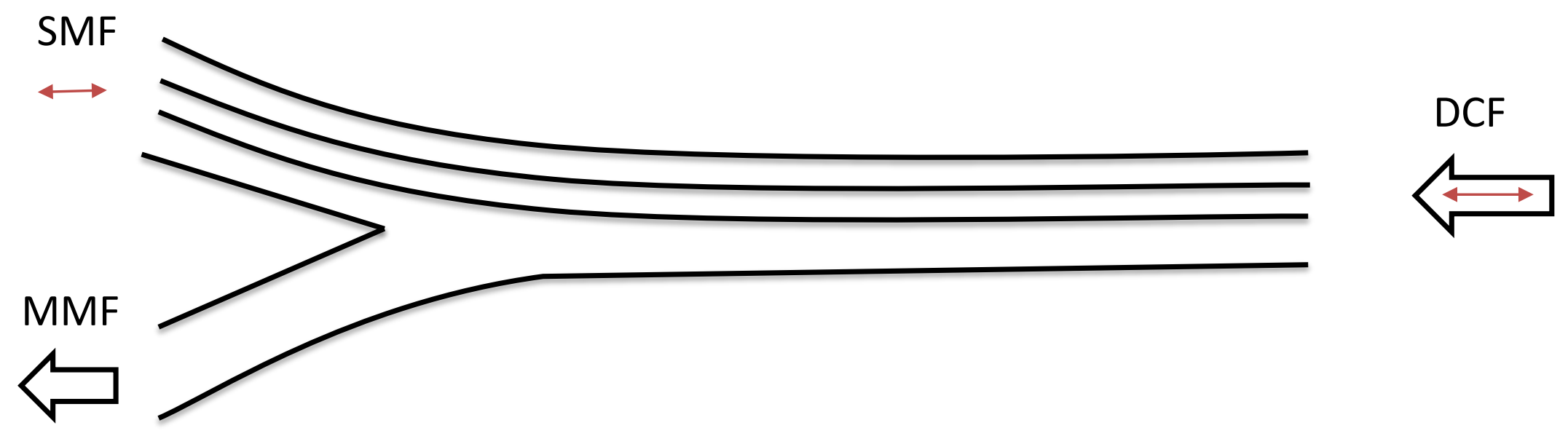

The DCF coupler separates the SM core signal from the MM inner cladding signal, enabling the separation of the transmitting signal from the received signal. 


\section{Modal Dispersion}

Different modes arrive at the end of the fiber at differing times due to different path lengths

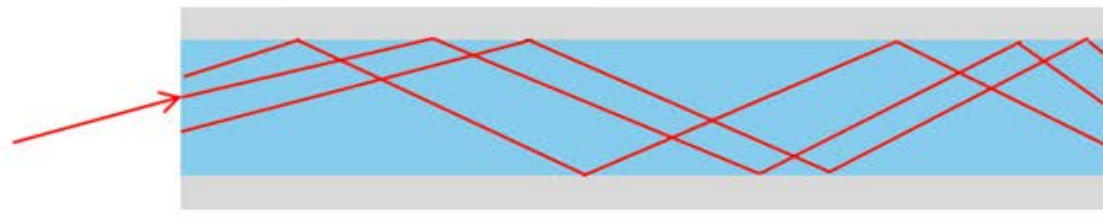

Multimode Fiber

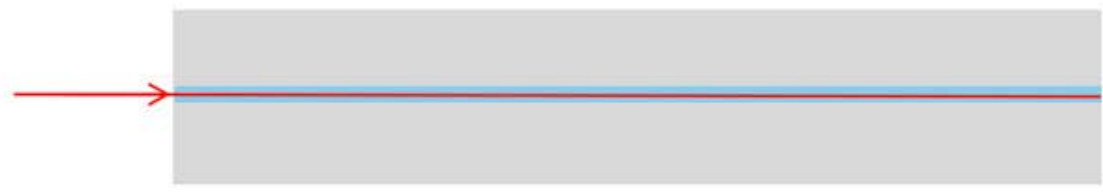

Sinale-Mode Fiber

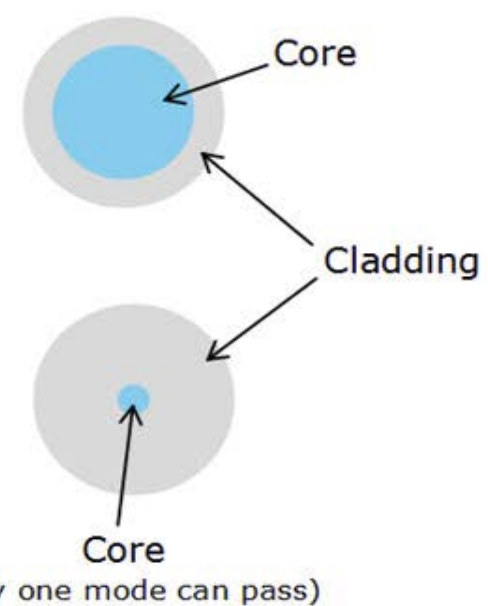

(Only one mode can pass)

This differential mode delay (modal dispersion) causes increases in bit error rates

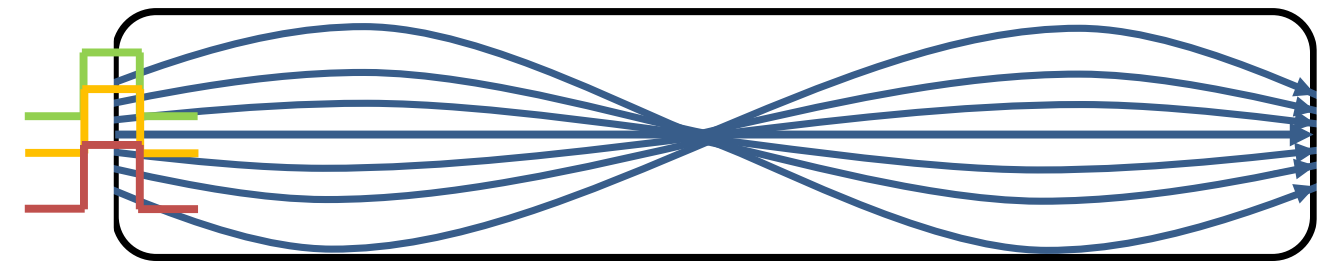




\section{BER Experimental Setup}

\section{Methods}

- Test combinations of single mode fibers (SMF), multi-mode fibers (MMF), and double clad fibers (DCF) in a FSOL setup at various divergence angles

- Tests will be run using vertical and horizontal misalignment

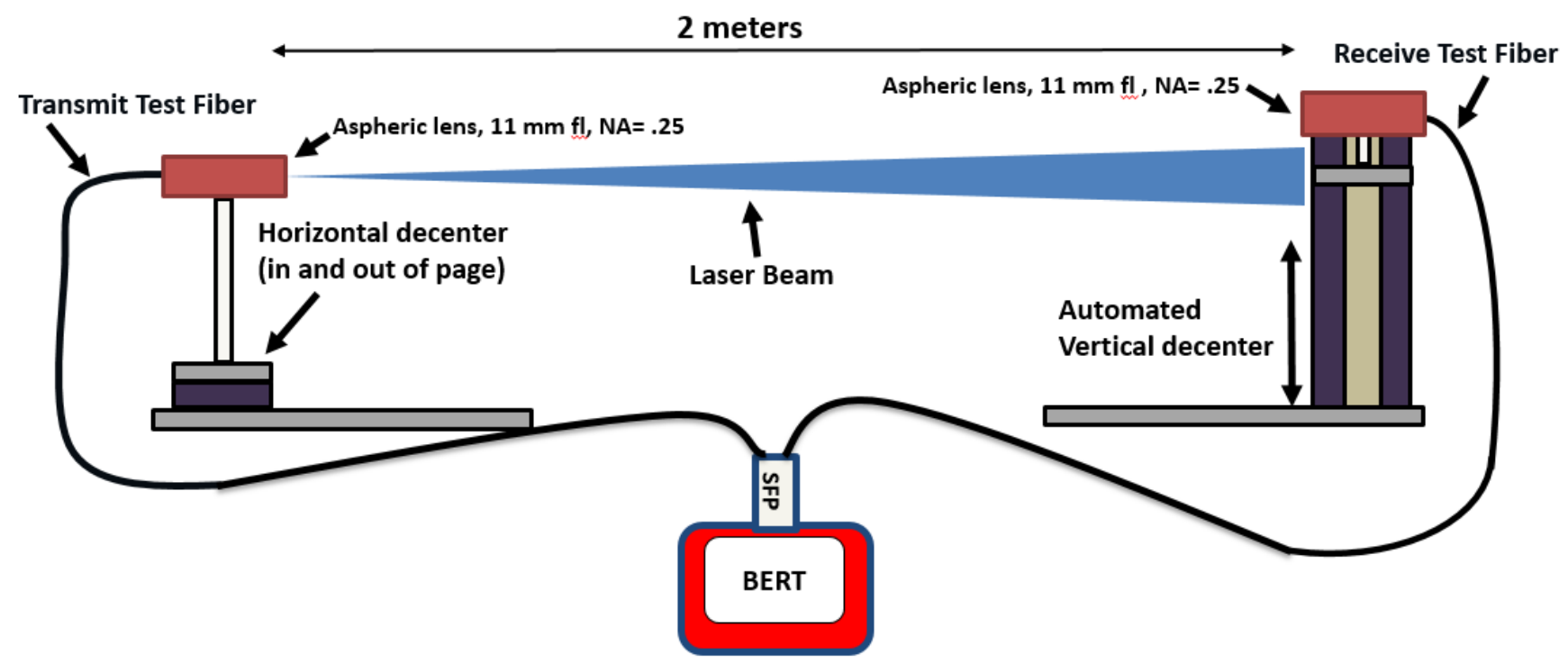

\begin{tabular}{|l|l|l|l|}
\hline Fiber & Core Size $($ um) & Graded or Step Index & Numerical Aperture \\
\hline Single Mode Fiber (SMF) & $9^{\mathrm{a}}$ & Graded & $0.12^{\mathrm{b}}$ \\
\hline Multimode Fiber (MMF) & 105 & Step & 0.22 \\
\hline Double Clad Fiber (DCF) & 9,105 & Step & $0.12,0.22$ \\
\hline \multicolumn{2}{|l}{ aMode Field Diameter. ${ }^{\mathrm{b}}$ Not Given, Typical Reported } & &
\end{tabular}




\section{Transmit Fiber Profiles}

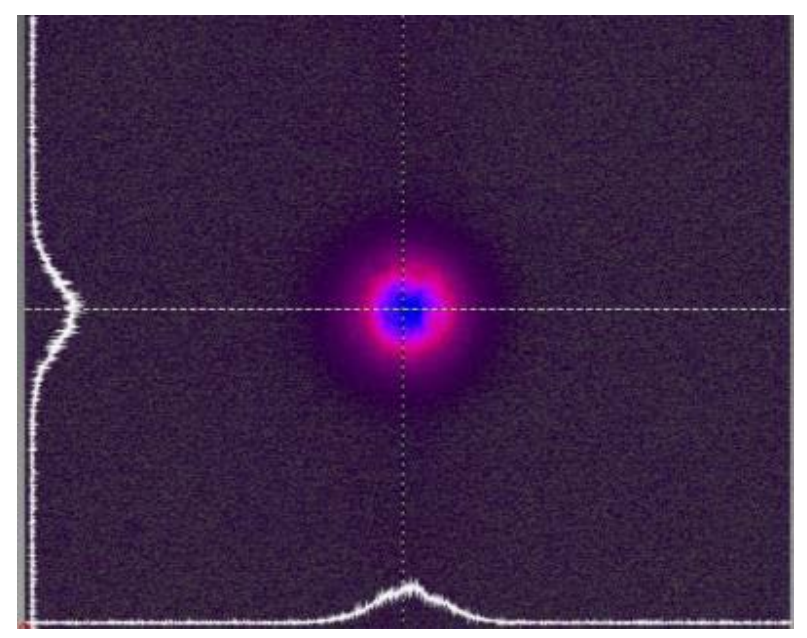

SMF

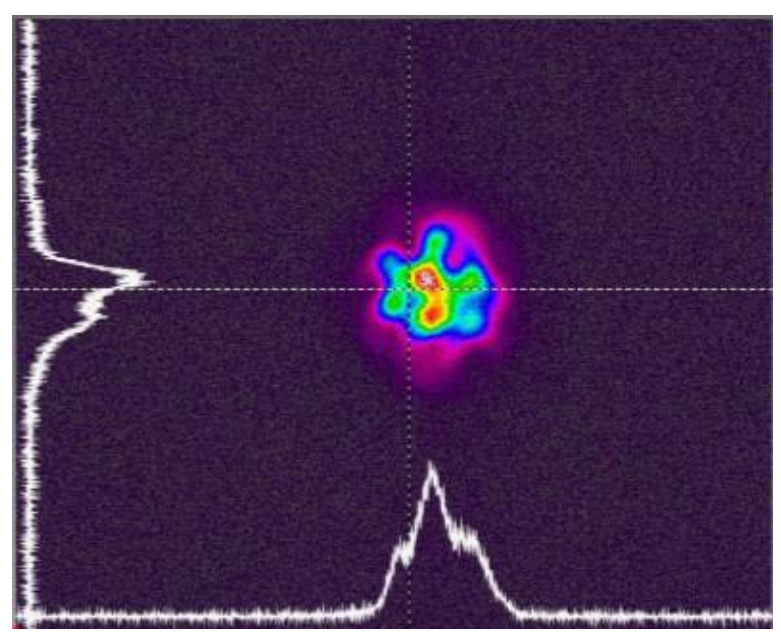

DCF

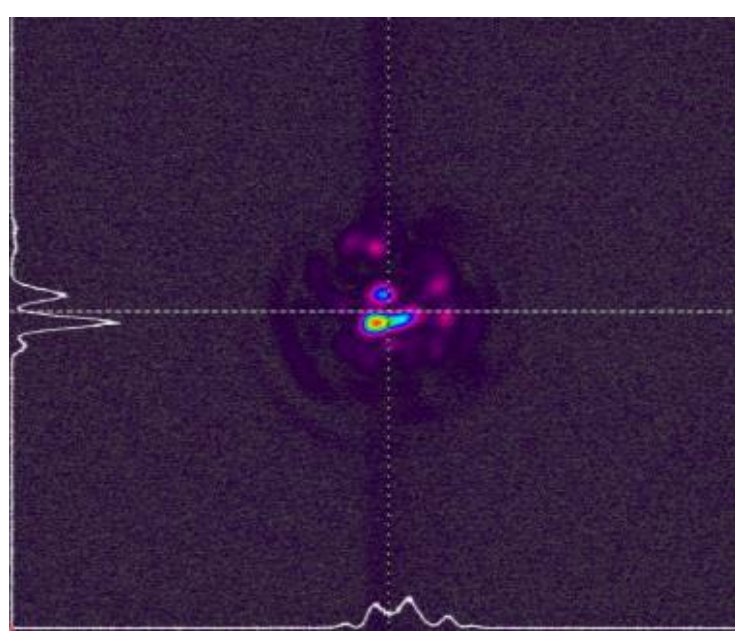

MMF

DCF as a transmitting fiber supports more modes than SMF, but fewer modes than MMF 

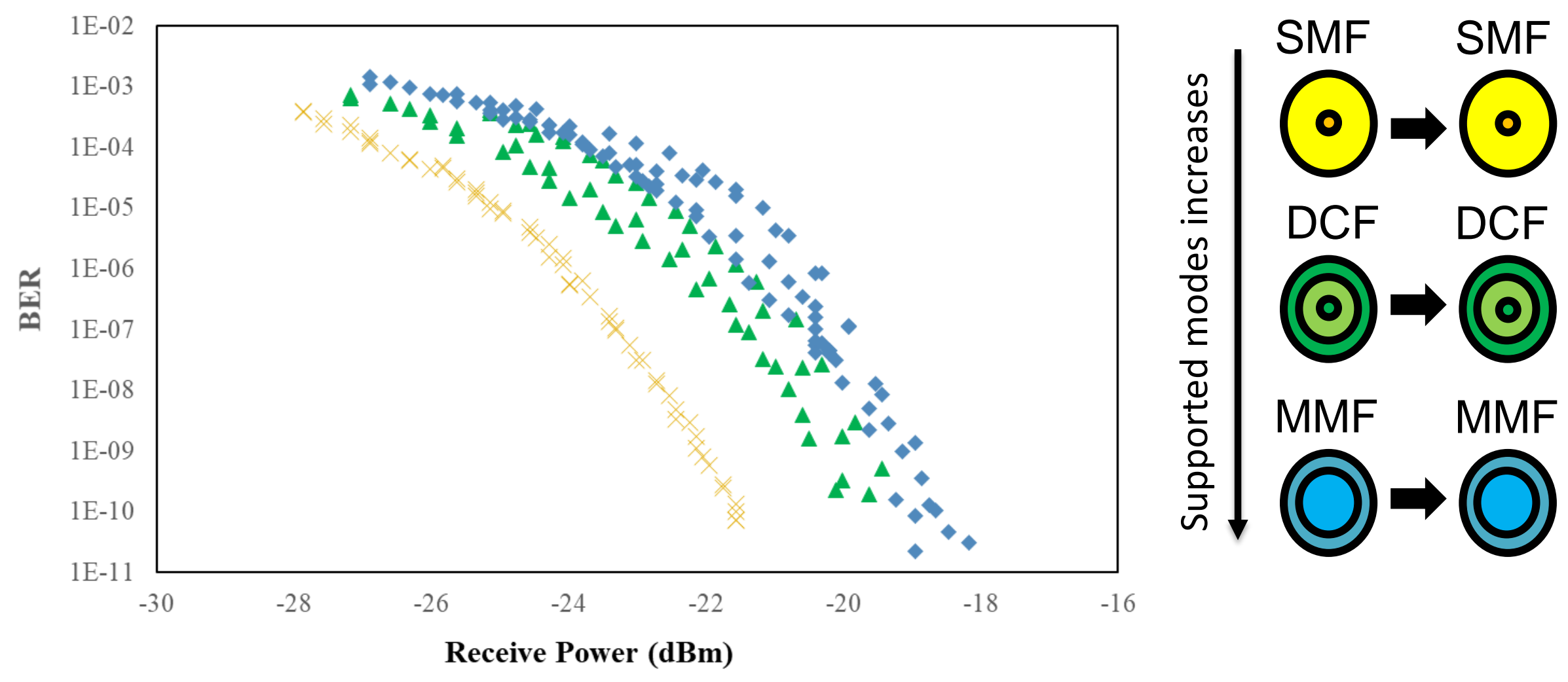

SMF-SMF $1.5 \mathrm{mrad} \quad$ DDCF-DCF $1.5 \mathrm{mrad} \quad$ MMF-MMF $1.5 \mathrm{mrad}$

BER increases as number of modes supported by the system increases 


\section{Misalignment Tolerance}

Lateral misalignment tolerance $($ Pointing Accuracy Tolerance $)=$ distance over which the BER is below $10^{-8}$

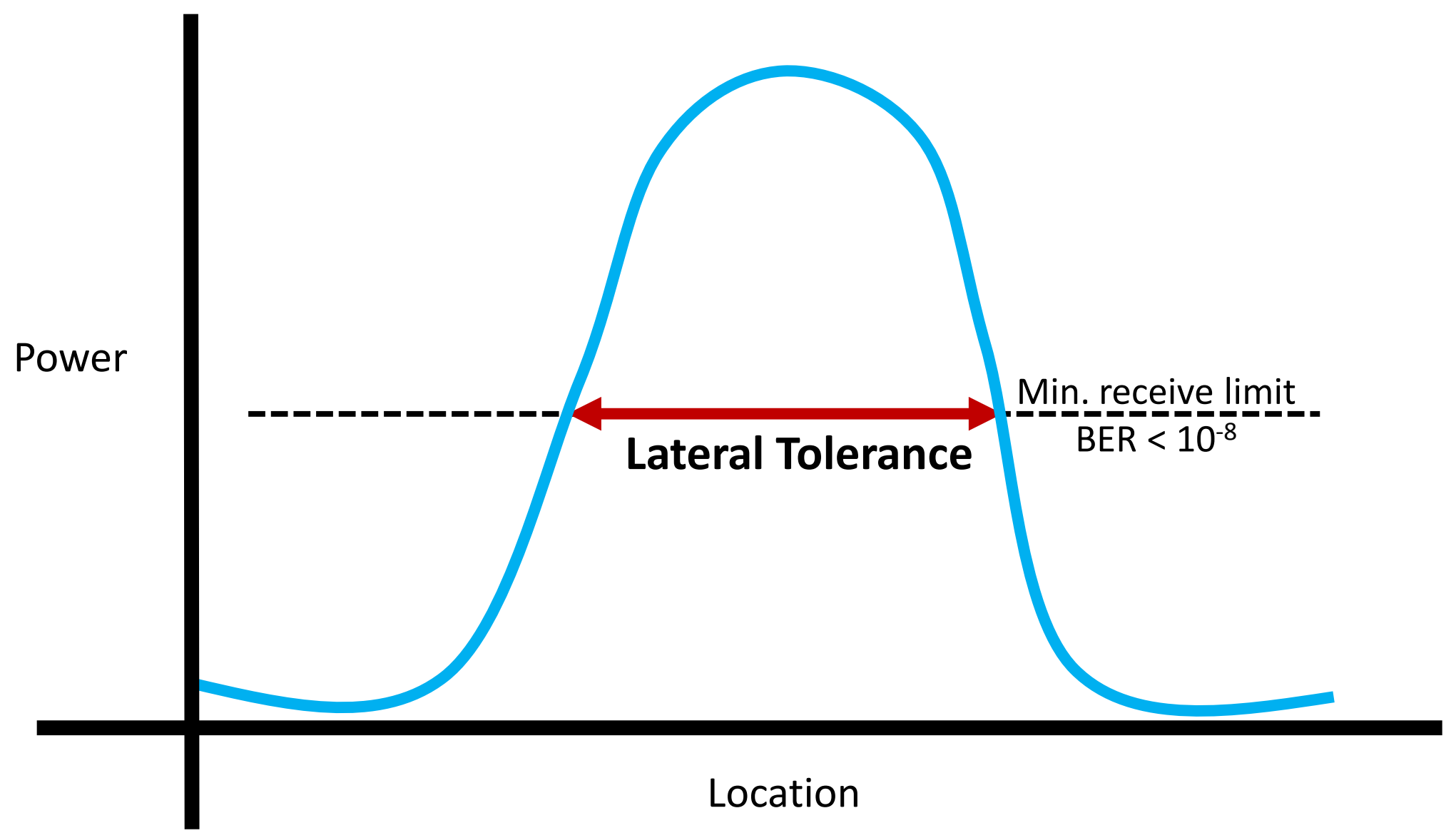




\section{Receive Fiber Results}
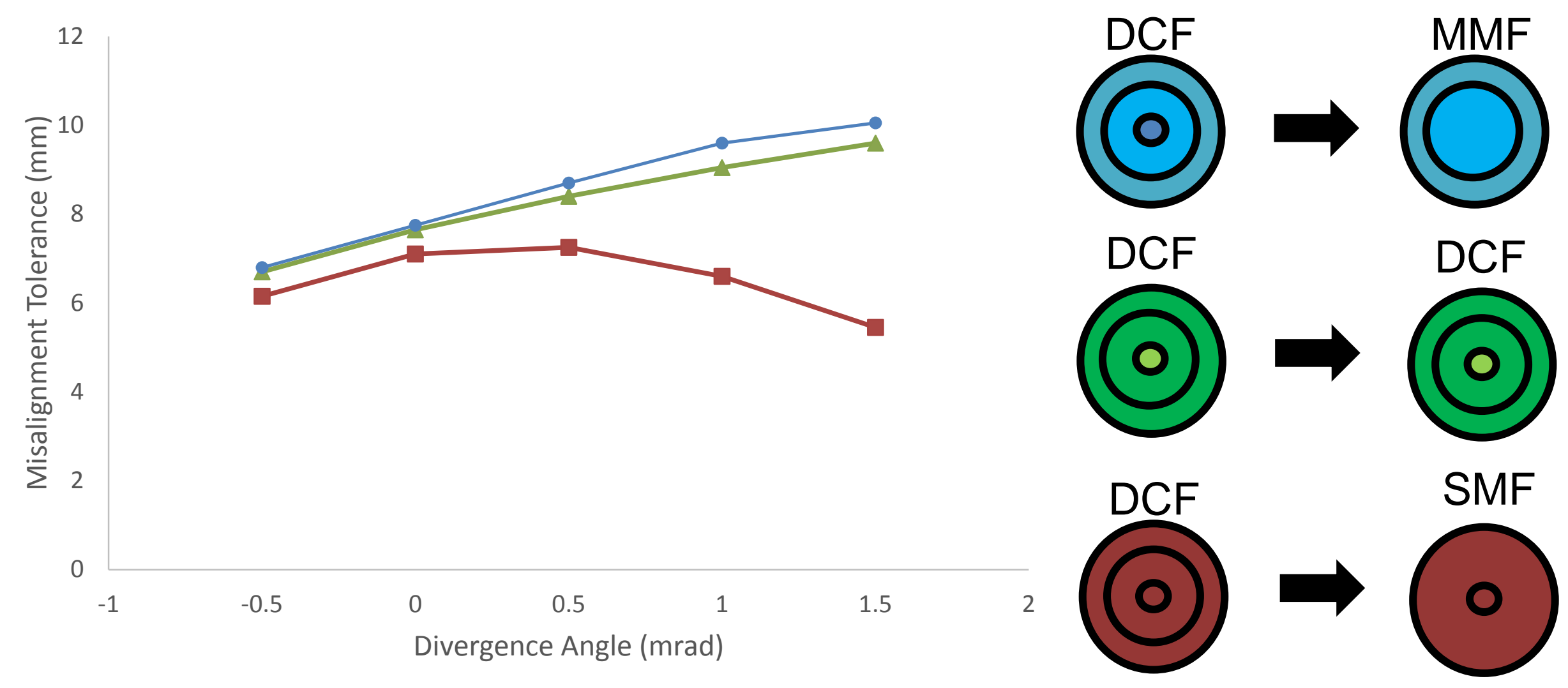

$\rightarrow$ DCF-DCF $\longrightarrow$ DCF-MMF DCF-SMF

DCF and MMF perform similarly as a receiving fiber. 


\section{Transmit Fiber Results}
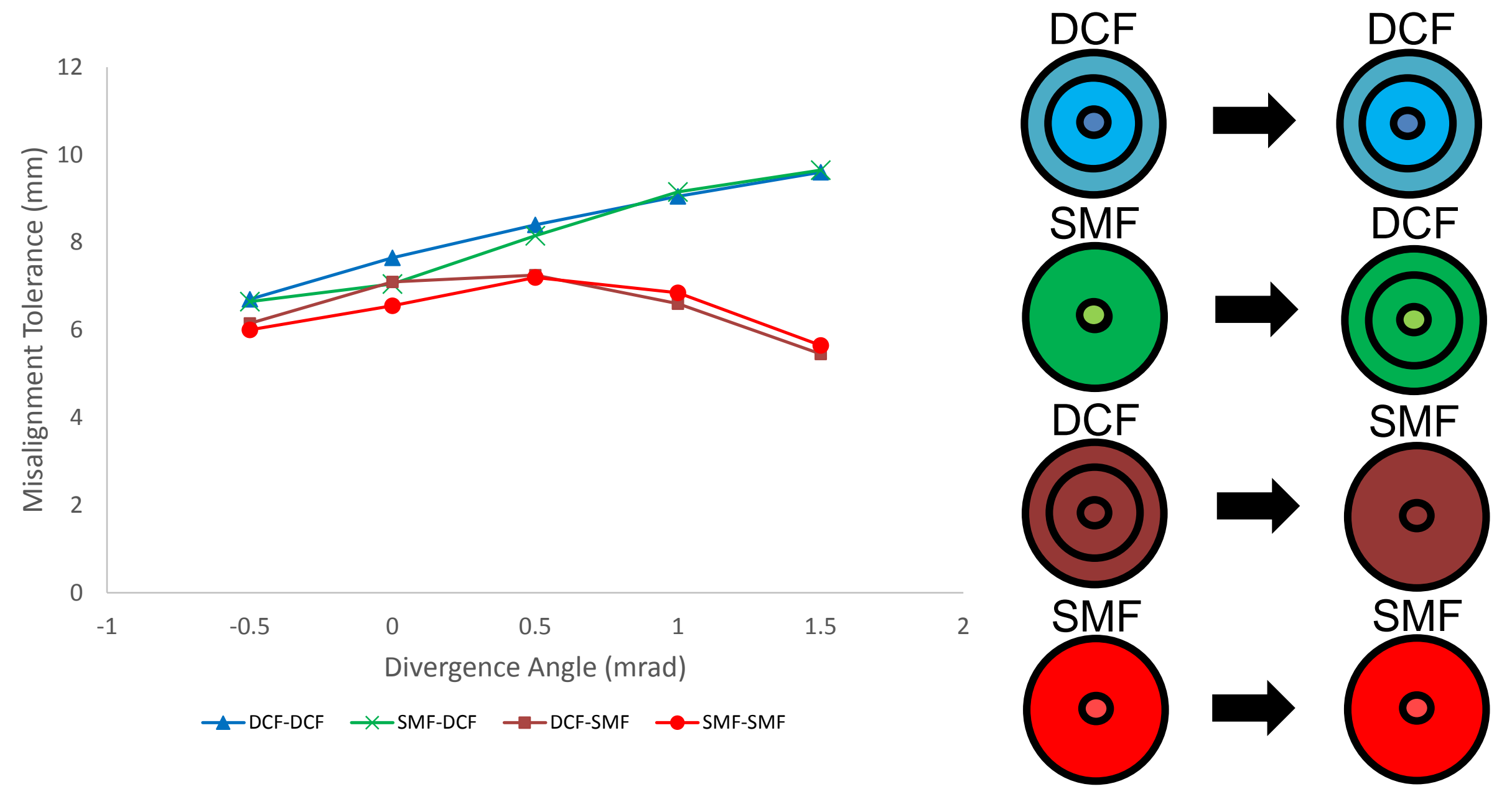

DCF and SMF have similar misalignment tolerance as transmitting fibers. 


\section{Transmit Fiber Results}
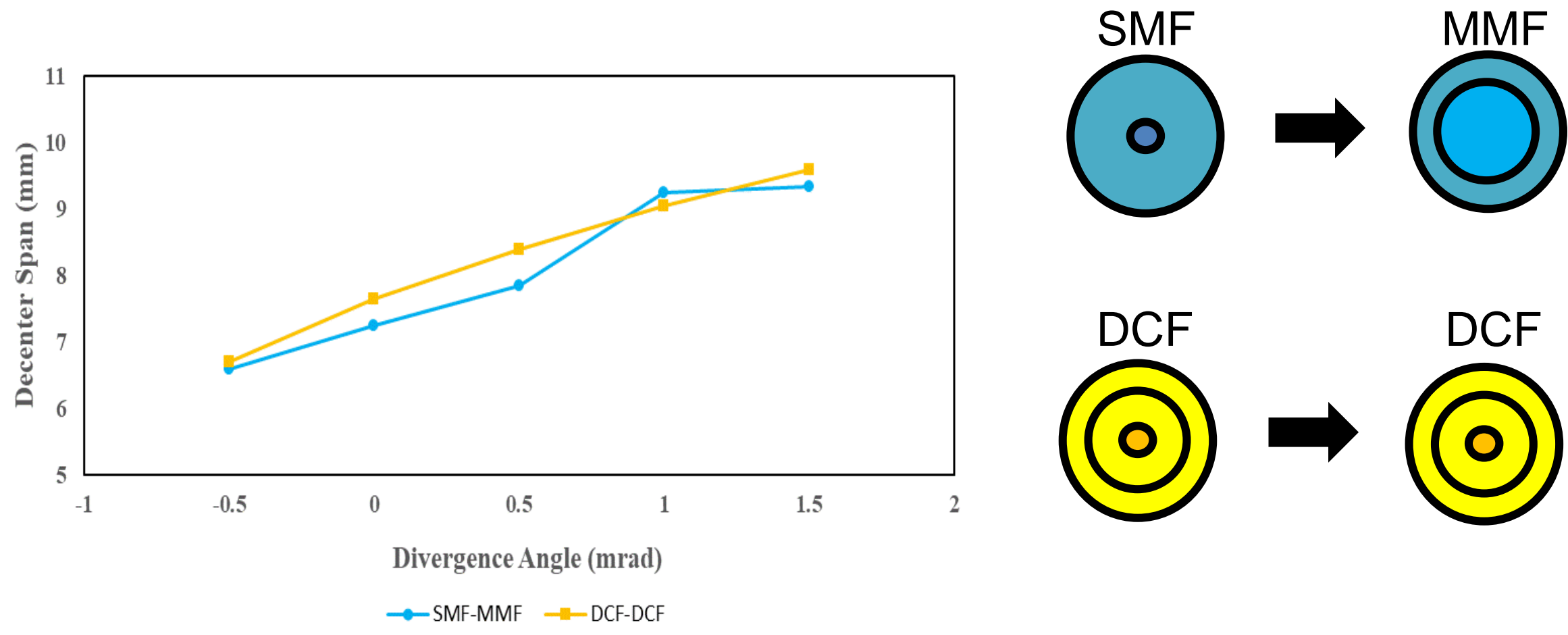

DCF-DCF has a similar misalignment tolerance to SMF-MMF, a common solution, and enables a symmetric setup. 


\section{NASA Launch Condition Experimental Setup}

2 meters

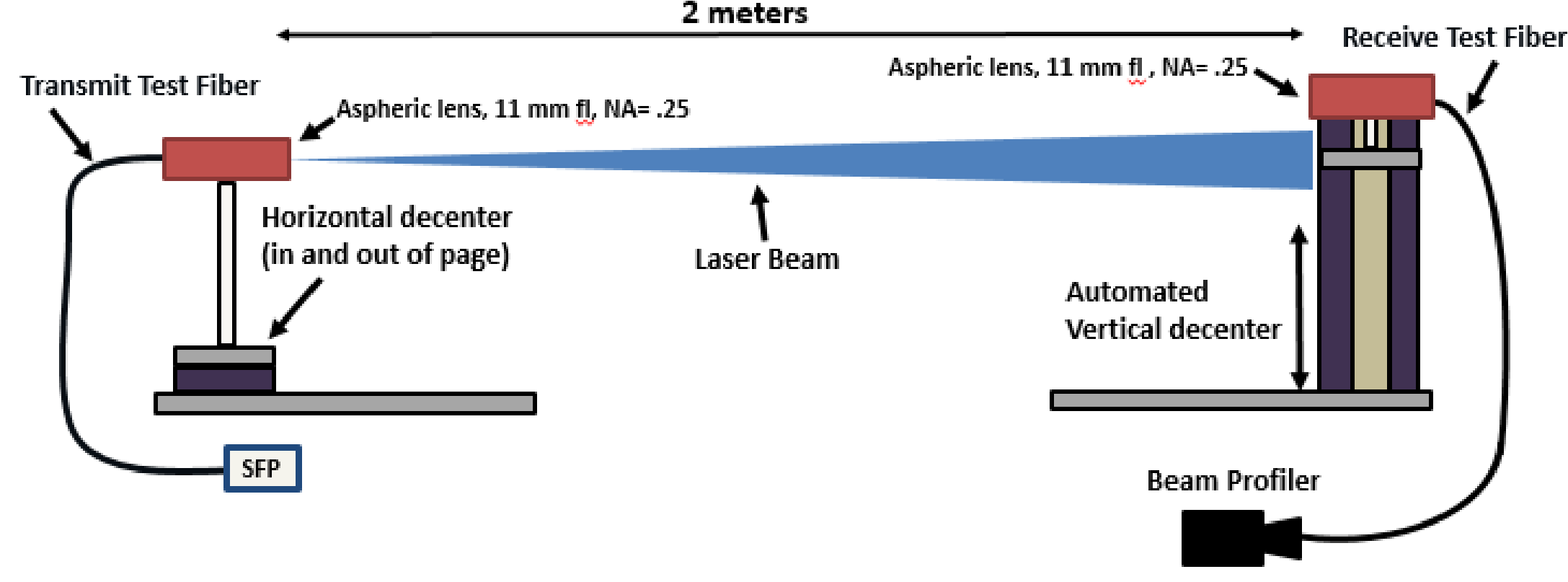

Beam Profiler was integrated into the setup to investigate the receive fiber launch conditions. 
 \\ Single Mode Fiber Rx Profile}

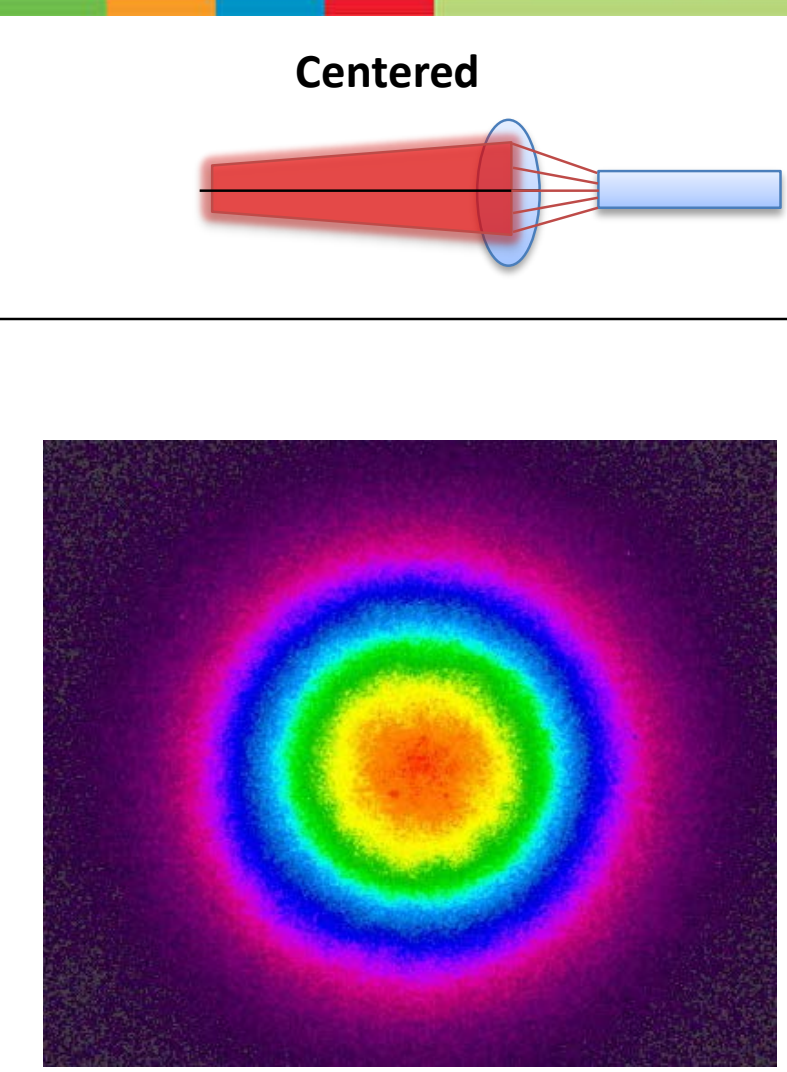

SMF - Centered

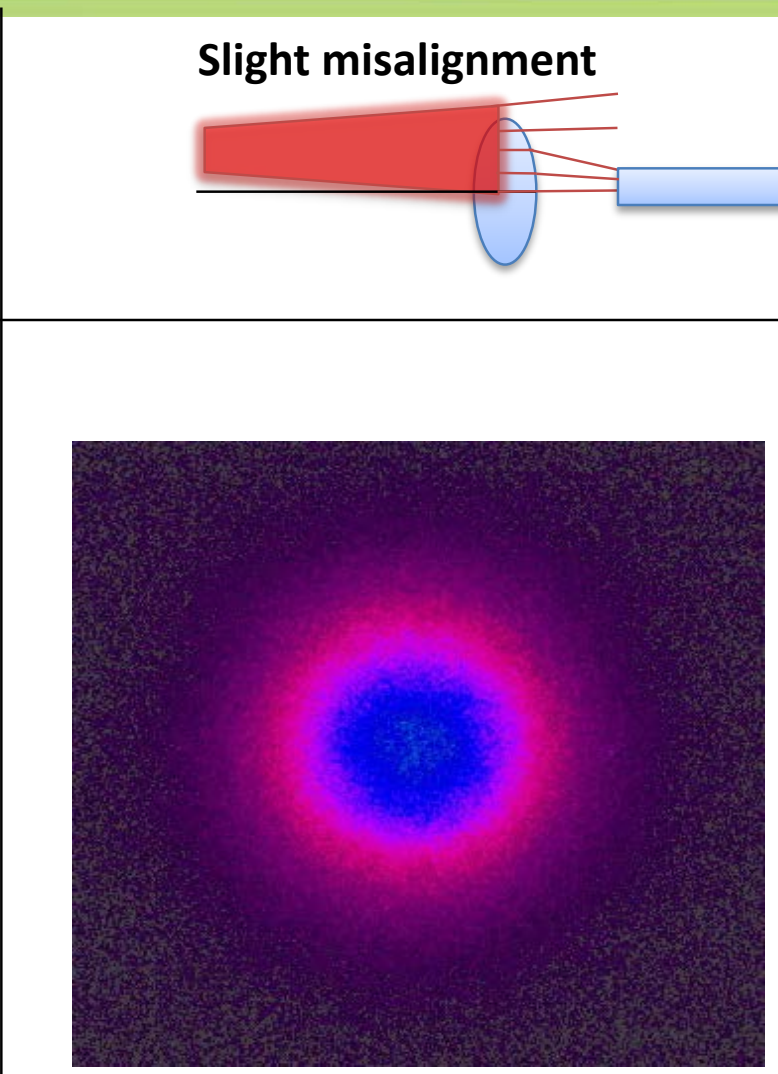

SMF - 2 mm decentered

\section{Extreme misalignment}

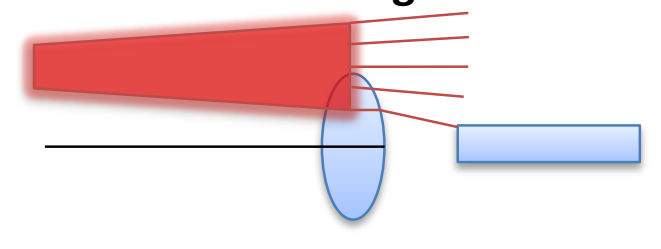

SMF -4 mm decentered

Power profile doesn't change as misalignment increases only a decrease in power is observed 


\section{Multi-Mode Fiber Rx Profile}

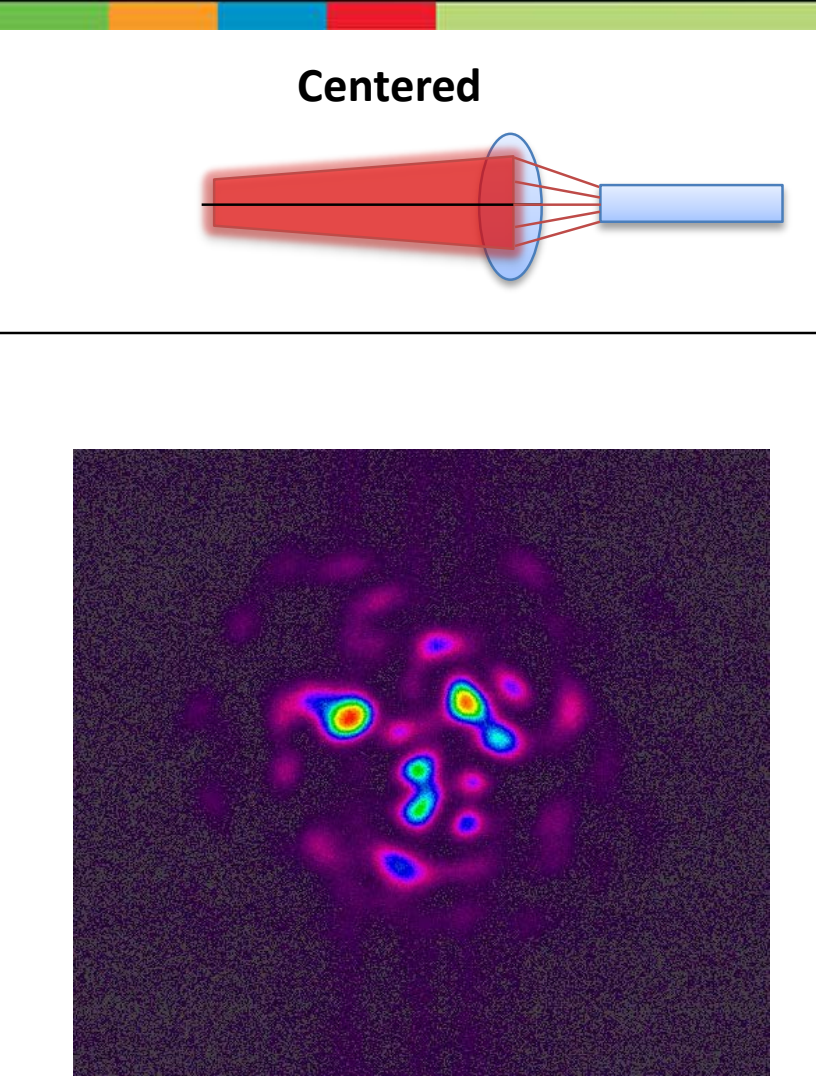

MMF - Centered

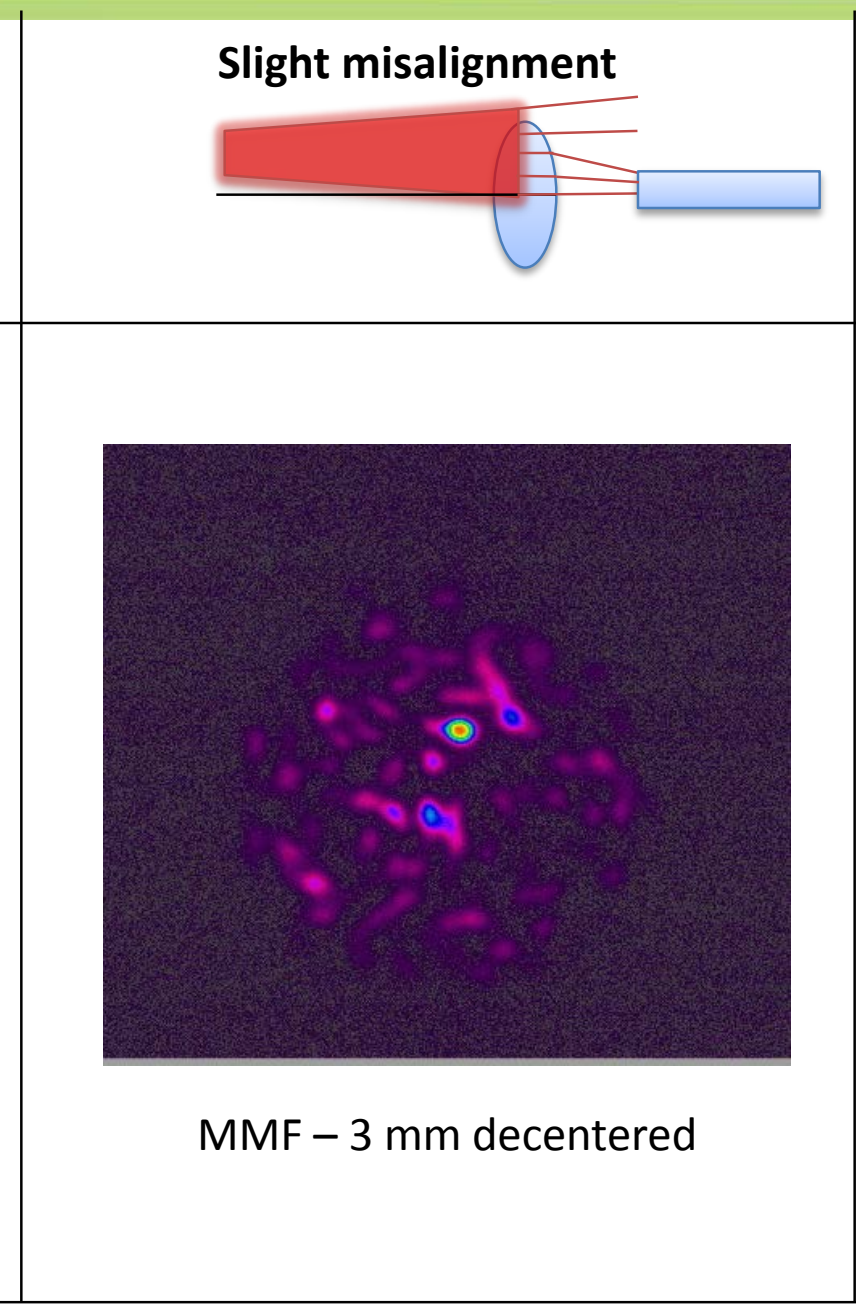

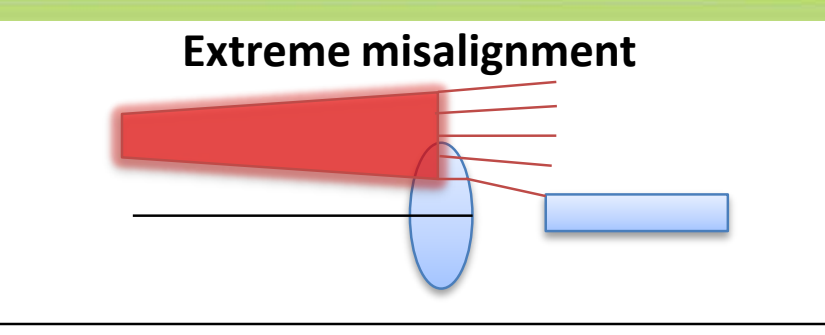

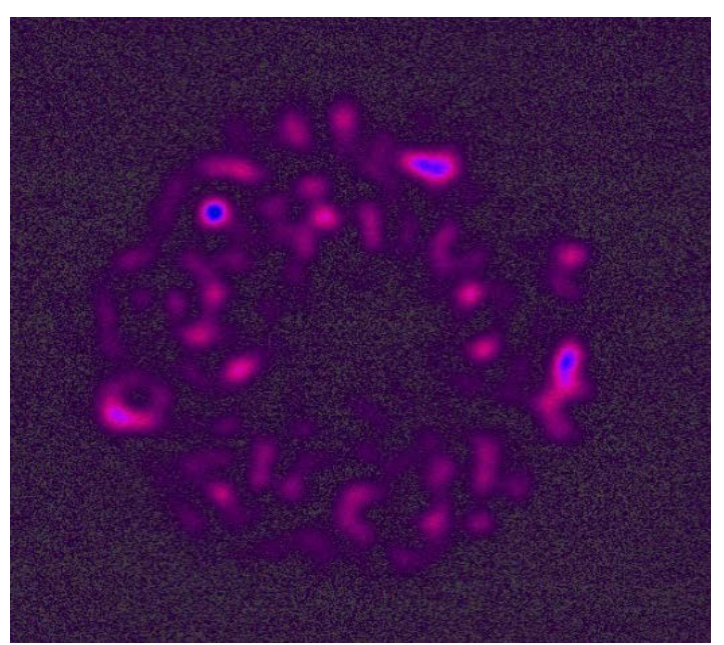

MMF - 5 mm decentered

Rx Power: $-28.73 \mathrm{dBm}$

Power moves outward radially as misalignment increases until skew rays dominate 


\section{Double Clad Fiber Rx Profile}

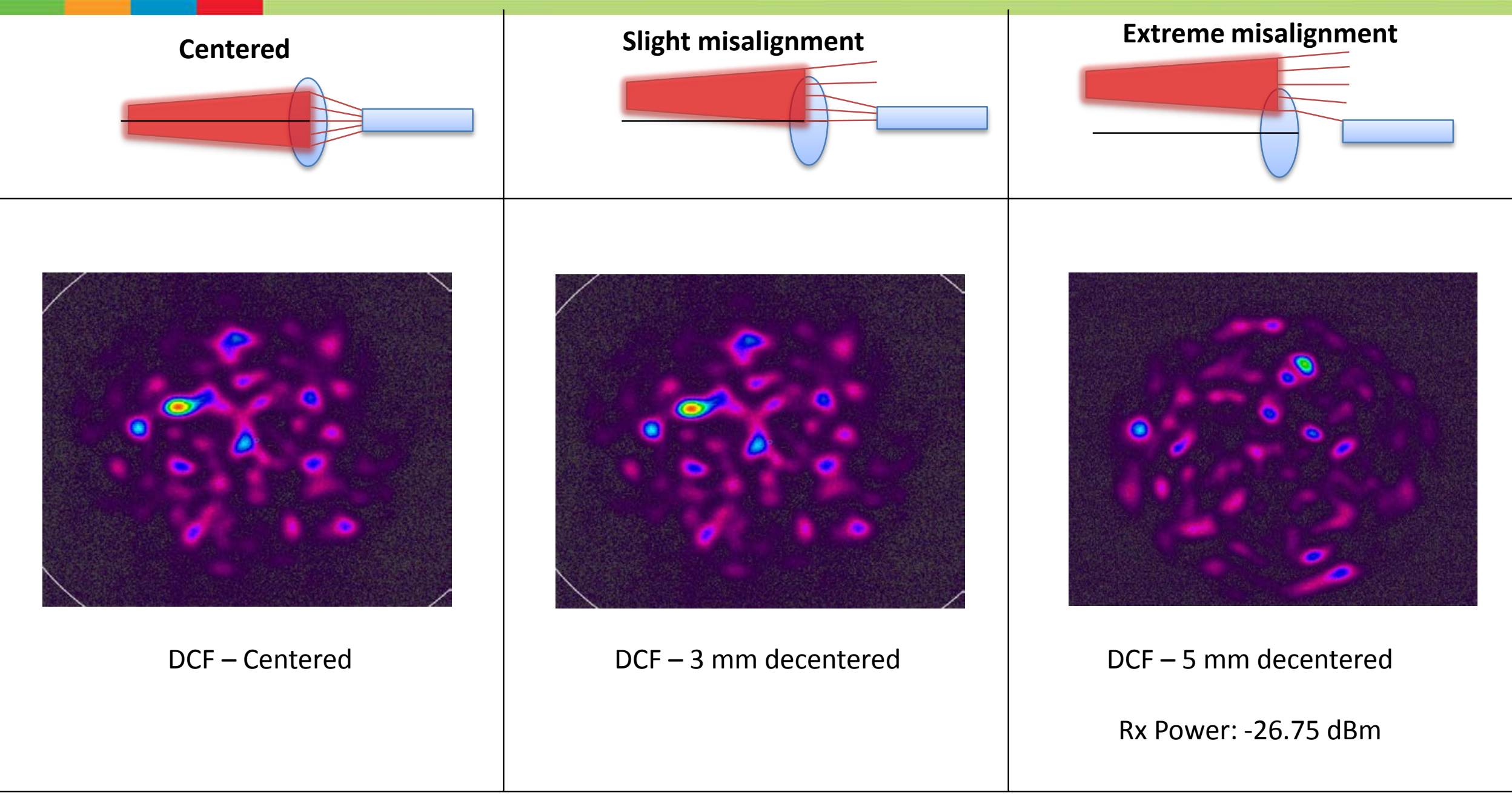

No skew rays observed in the DCF for same conditions 


\section{Conclusion}

\section{Summary}

- Findings demonstrate the viability of a low-SWaP, bidirectional, symmetric FSOL utilizing DCF to transmit and receive.

- The BER misalignment performance of the DCF was comparable to the SMF while transmitting, and to the MMF while receiving.

- Skew rays were observed in the MMF, but not in the DCF, resulting in receive power losses.

\section{Future Work}

- Quantify the effect of skew rays on the BER performance

- Determine if/when skew rays are present in DCF using higher transmit power

- Setup and test a symmetric duplex FSOL using DCF.

\section{Acknowledgements}

- This work was funded by Space Communications and Navigation Program at NASA.

MMF - 5 mm decentered
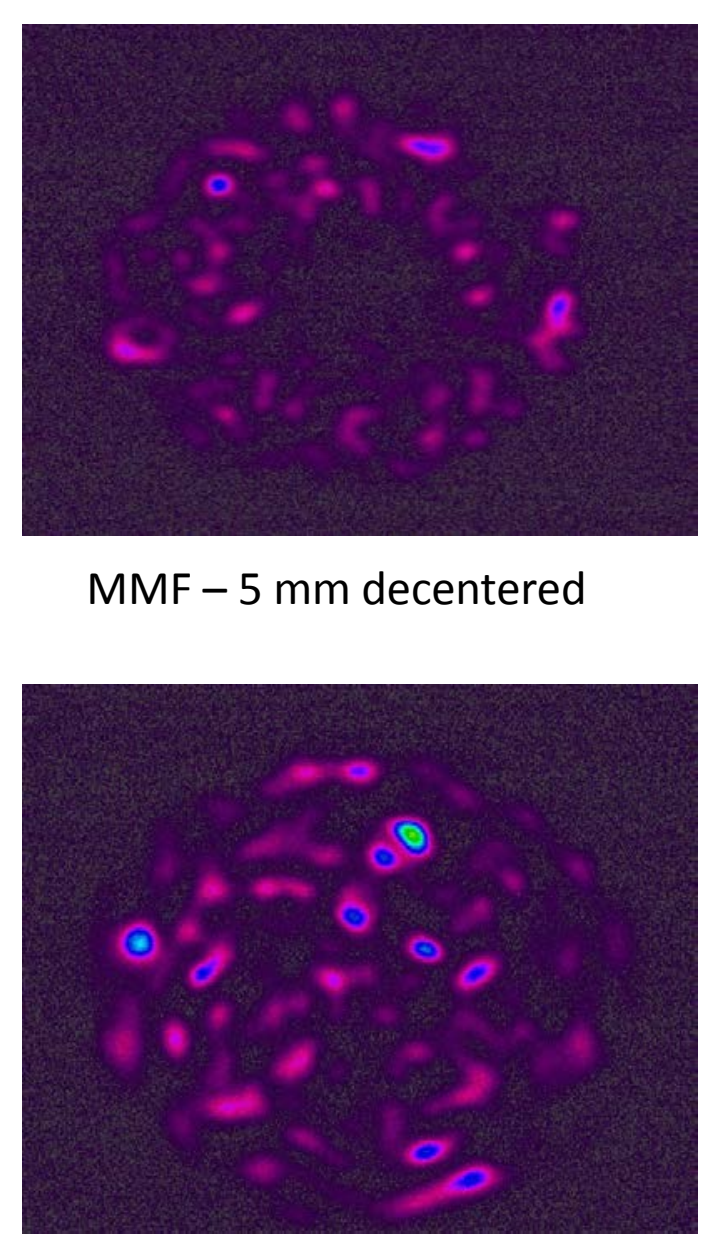

DCF $-5 \mathrm{~mm}$ decentered 